\title{
Rendimiento, equidad y calidad: el desarrollo educativo en España según PISA 2015*
}

\author{
Antonio Villar \\ Universidad Pablo de Olavide e IVIE
}

\section{Resumen}

Este trabajo analiza los resultados de PISA 2015 en España teniendo en cuenta tres dimensiones diferentes de los resultados educativos: el rendimiento (valores medios de los test), la equidad (el grado de dependencia de los resultados de los estudiantes de su entorno familiar) y la calidad (las proporciones de estudiantes de alto y bajo rendimiento). Los resultados muestran que existen diferencias importantes en los resultados educativos, que no se reflejan adecuadamente en la mera comparación de los valores medios de los test.

Palabras clave: PISA 2015, España y sus comunidades autónomas, desarrollo educativo, rendimiento, equidad, calidad.

Clasificación JEL: I21, I24.

\begin{abstract}
This paper analyses the results in the PISA 2015 report in Spain and its autonomous regions. It focuses on three different dimensions: performance (average scores of the test), equity (dependence of those outcomes on the family environment) and quality (shares of high and low performers). The outcomes show that there exist much wider differences between the regions than those captured by the average scores.
\end{abstract}

Keywords: PISA 2015, Spain and its autonomous regions, educational development, performance, equity, quality.

JEL classification: I21, I24.

\section{Introducción}

El capital humano es sin duda uno de los determinantes esenciales del progreso de las sociedades. La formación reglada y la experiencia adquirida mediante la actividad profesional son los componentes básicos de ese capital humano. De hecho, los niveles formativos de la población en edad de trabajar son una referencia clave a la hora de explicar el grado de desarrollo de las sociedades (Acemoglou y Robinson, 2013).

* Este trabajo se enmarca en una línea de investigación financiada por el Ministerio de Ciencia e Innovación (proyecto ECO2015-65408-R MINECO/FEDER/UE). Los comentarios y sugerencias de un evaluador anónimo han permitido mejorar la redacción del mismo. 
Desde el año 2000, la Organización para la Cooperación y el Desarrollo Económico (OCDE) viene realizando estudios sobre las capacidades adquiridas por los estudiantes de 15 años en tres distintas materias: comprensión lectora, matemáticas y ciencias. Se trata de los conocidos como «informes PISA», acrónimo de Programme for the International Students Assessment. El objeto de estos estudios, de carácter trianual, que en cada ocasión toman como eje central una de las tres materias indicadas, es evaluar las capacidades de los estudiantes que están próximos a terminar la educación obligatoria. En 2015, el informe PISA se centró en el área de Ciencias.

Hay varios aspectos que hacen de estos informes una fuente de información especialmente relevante sobre los sistemas educativos y la formación del capital humano. En primer lugar porque valoran los resultados educativos en términos de las capacidades efectivamente adquiridas y no en términos de aspectos formales (años cursados, titulaciones obtenidas o contenidos curriculares). En segundo lugar, y en parte como consecuencia de lo anterior, porque este estudio permite hacer comparaciones internacionales en términos de estándares comunes. En tercer lugar porque la edad tomada como referencia para la evaluación corresponde al final de la etapa de la enseñanza obligatoria. Ello implica que estos informes nos proporcionan una aproximación sobre cuáles son los mínimos niveles de conocimiento que cada sociedad garantiza a sus miembros. En cuarto lugar porque la periodicidad de su realización permite analizar no solo la situación en un determinado momento, sino también la evolución de los sistemas educativos. Por último, y no menos importante, porque además de realizar los test de conocimientos, los alumnos completan un exhaustivo cuestionario relativo a su entorno familiar, sus hábitos de estudio, sus actitudes y su motivación. Ello permite relacionar los resultados obtenidos con las variables económicas, demográficas y sociales y poder interpretar así las diferencias observadas para poder diseñar políticas de mejora.

En resumen, los Informes PISA proporcionan un rico perfil de las habilidades y conocimientos de los alumnos de 15 años, así como una valiosa información contextual que puede servir de base para el diseño de políticas de mejora.

El elemento central que articula la medición del conocimiento de los estudiantes de 15 años es la puntuación obtenida en un test elaborado de forma que permita las comparaciones internacionales. Para cada región o país se calcula la media de las puntuaciones obtenidas por los estudiantes (una muestra representativa para cada región o país considerado) ${ }^{1}$. Se fijó una escala de 1 a 1.000 con una media de 500 y una desviación típica de 100 en el año 2000. Esos puntos de referencia dan significado a las cifras obtenidas por los distintos países. A partir de estos valores medios se elaboran los rankings que luego se presentan en los medios de comunicación. Conviene recordar, sin embargo, que el fin último de estos informes no es hacer un ranking de países como si fuera una competición deportiva, sino proporcionar información que permita diseñar medidas de mejora para los diferentes sistemas educativos.

\footnotetext{
${ }^{1}$ En realidad no se trata de una media simple, ya que el informe recurre al uso de los denominados «valores plausibles» que permiten generar intervalos de confianza para las observaciones individuales.
} 
Setenta y dos países y grandes economías participaron en PISA 2015. Por primera vez todas las comunidades autónomas españolas ampliaron las muestras de estudiantes con objeto de tener resultados representativos a nivel regional.

El objeto de este trabajo es proporcionar una panorámica sobre algunos de los aspectos centrales del informe PISA 2015 en relación con España y sus comunidades autónomas. Nos centraremos en al análisis de tres variables que miden distintos aspectos de los resultados que ofrecen los sistemas educativos: el rendimiento, la equidad y la calidad. El rendimiento se asocia a los valores medios del test en cada comunidad. La equidad se aproxima mediante una variable que mide el grado de dependencia de los resultados educativos del origen familiar. Y la calidad se asocia al porcentaje de los estudiantes de alto y bajo rendimiento (las colas de la distribución). Siguiendo una idea desarrollada en Villar (2013) estas tres variables se integran en un indicador conocido como «índice de desarrollo educativo».

\section{Rendimiento, equidad y calidad}

Hay una primera observación importante que debemos tener en cuenta a la hora de interpretar adecuadamente los resultados de los Informes PISA (Programa Internacional de Evaluación de los Alumnos, por sus siglas en inglés): la comparación de los valores medios de los test es muy poco informativa, porque las distribuciones de resultados son muy distintas en los diferentes países y regiones. Solo a partir del estudio de las distribuciones se pueden diseñar políticas de mejora. El Gráfico 1 ilustra bien estas diferencias en las distribuciones. Recoge las distribuciones de cuatro países: Singapur y República Dominicana, que representan los mejores y los peores resultados medios de los países participantes, y España y Estados Unidos. Estos dos últimos tienen medias similares, pero distribuciones sustancialmente distintas en las que se aprecia que la cola derecha (alto rendimiento) es más robusta en el caso de Estados Unidos.

Comparar distribuciones completas es un ejercicio extremadamente complejo desde el punto de vista técnico y no siempre posible. Una forma simplificada de tomar en cuenta las diferencias en las distribuciones de resultados es analizar las proporciones de estudiantes en los seis (en realidad siete) «niveles de rendimiento» que establece la OCDE, parametrizados por umbrales del test tal y como refleja el Cuadro 1.

De acuerdo con el Informe PISA España (Ministerio de Educación, Cultura y Deporte, 2016), «Los factores utilizados para determinar la exigencia cognitiva o grado de dificultad de las preguntas que evalúan el rendimiento en ciencias incluyen:

- el número y el grado de complejidad del conocimiento en que se centra la pregunta;

- el nivel de familiaridad y conocimiento previo que los estudiantes puedan tener de los contenidos, procedimientos y actitudes en relación con la pregunta; 


\section{GRÁFICO 1 \\ DISTRIBUCIONES DE RESULTADOS DEL TEST PISA 2105 (CIENCIAS) PARA ALGUNOS PAÍSES SELECCIONADOS}

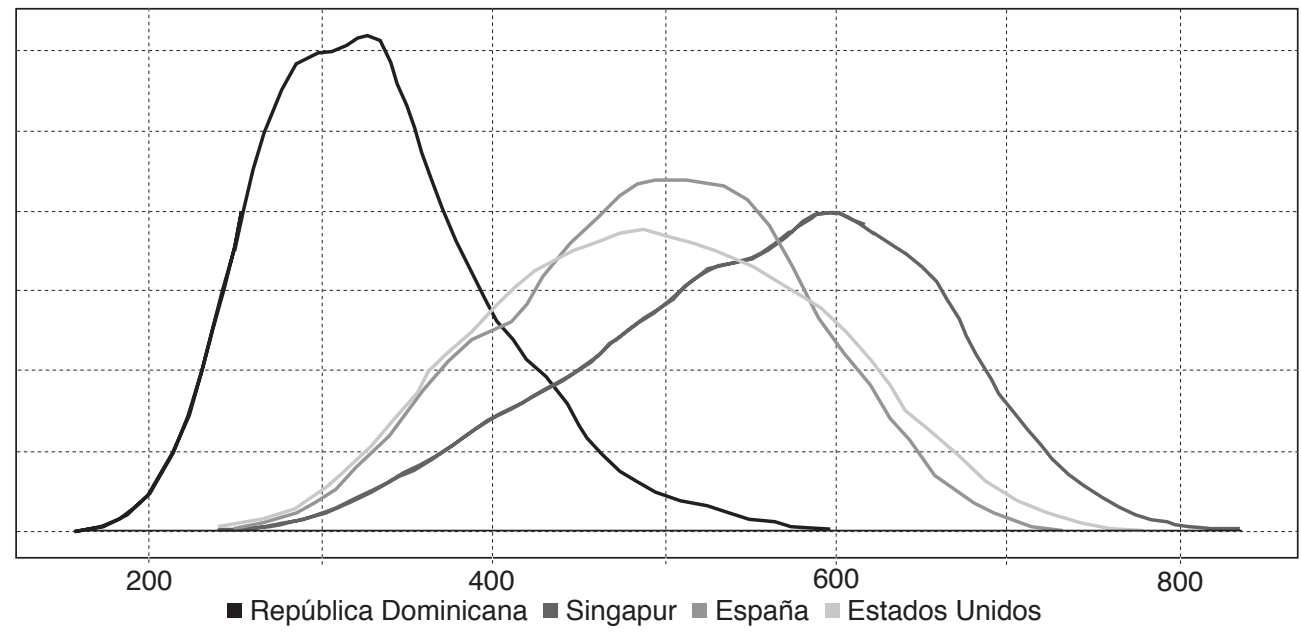

FUENTE: OCDE.

\section{CUADRO 1}

RANGO DE VALORES DEL TEST PARA LOS DISTINTOS NIVELES DE RENDIMIENTO (PISA 2015, CIENCIAS)

\begin{tabular}{|c|c|}
\hline Nivel de rendimiento & Rango de puntuación \\
\hline Nivel 1b & {$[0,335)$} \\
\hline Nivel 1a & {$[335,410)$} \\
\hline Nivel 2 & {$[410,484)$} \\
\hline Nivel 3 & {$[484,559)$} \\
\hline Nivel 4 & {$[559,663)$} \\
\hline Nivel 5 & {$[663,708)$} \\
\hline Nivel 6 & $>708$ \\
\hline
\end{tabular}

FUENTE: OCDE.

- el funcionamiento cognitivo requerido por la pregunta, por ejemplo, memoria, análisis, valoración;

- el grado en que la formación de una respuesta depende de modelos o ideas científicas abstractas.»

De estos niveles de rendimiento hay dos que se consideran como puntos de referencia especialmente significativos, el nivel 2 y el nivel 5, porque marcan lo que por convención se considera bajo y alto rendimiento. Aquellos estudiantes por debajo 
del nivel 2, niveles 1a y $1 \mathrm{~b}$, son considerados estudiantes de bajo rendimiento (low performers), mientras que aquellos estudiantes con niveles de rendimiento 5 y 6 son considerados como de alto rendimiento (high performers).

Según el Informe PISA, «En el nivel 2, el alumno es capaz de usar conocimiento sustantivo de la vida diaria y conocimiento procedimental básico para identificar una explicación científica, interpretar datos e identificar la pregunta a la que responde un diseño experimental sencillo. Puede describir relaciones causales simples. Demuestra un conocimiento epistémico elemental al ser capaz de identificar preguntas que se pueden investigar científicamente» (Ministerio de Educación, Cultura y Deporte 2016, p. 64). El nivel 2 es así considerado como el mínimo nivel de rendimiento que permite a los individuos una inserción en la vida económica y social con perspectivas razonablemente positivas. Hay evidencia que indica que por debajo de ese nivel los individuos tendrán dificultades de integración en el mundo sociolaboral.

«En el nivel 5, el alumno es capaz de utilizar conocimiento de contenido sustantivo, procedimental y epistémico para explicar fenómenos no familiares y complejos, sucesos y procesos con cadenas causales jerarquizadas y múltiples. Son capaces de aplicar un conocimiento epistémico bastante sofisticado para evaluar diseños experimentales alternativos, justificar su elección y usar su conocimiento teórico para interpretar información y hacer predicciones. Al nivel 5, el alumno puede evaluar formas de explorar científicamente una pregunta dada e identificar las limitaciones en interpretaciones de conjuntos de datos incluyendo fuentes y los efectos de la incertidumbre de los datos científicos» (ibid.).

El Cuadro 2 permite situar a España en el contexto de la OCDE a partir de los resultados del informe PISA 2015 en el ámbito de ciencias. Proporciona información tanto sobre los valores medios de los test como sobre los porcentajes de estudiantes con alto y bajo rendimiento. La primera observación a destacar es la de que la principal variabilidad entre los países se encuentra en las colas de la distribución y no en los valores medios: el coeficiente de variación $(\mathrm{CV})$ de la variable estudiantes con bajo rendimiento es 7,3 veces el del valor medio del test (puntuación) y la variable estudiantes con alto rendimiento tiene un $\mathrm{CV}$ nueve veces el del valor medio.

España presenta una puntuación que coincide con la media de la OCDE, ligeramente por debajo de la media de la Unión Europea, que se sitúa en 495 puntos. El porcentaje de estudiantes españoles que presentan alto rendimiento supone un 65 por 100 de la media de la OCDE, lo que indica un claro déficit en este segmento. Nuestros datos son mejores que la media de la OCDE en cuanto al porcentaje de estudiantes con bajo rendimiento (un 86 por 100 de dicha media). Australia, Canadá, Estonia, Finlandia, Alemania, Japón, Corea, Holanda, Nueva Zelanda y Reino Unido presentan porcentajes de estudiantes de alto rendimiento que más que duplican el porcentaje de los que tiene España. Todos ellos presentan además porcentajes de estudiantes con bajo rendimiento inferiores a España (con la excepción de Holanda, que tiene un valor similar).

El Gráfico 2 proporciona una primera visión de la situación de las comunidades autónomas españolas en relación con estos niveles críticos de referencia. La simple 


\section{CUADRO 2}

RENDIMIENTO EN CIENCIAS EN LOS PAÍSES DE LA OCDE

\begin{tabular}{|c|c|c|c|}
\hline & Puntuación & $\begin{array}{l}\text { \% estudiantes bajo } \\
\text { rendimiento }\end{array}$ & $\begin{array}{l}\% \text { estudiantes alto } \\
\text { rendimiento }\end{array}$ \\
\hline Alemania & 509 & 17,0 & 10,6 \\
\hline Australia & 510 & 17,6 & 11,2 \\
\hline Austria & 495 & 20,8 & 7,7 \\
\hline Bélgica & 502 & 19,8 & 9,0 \\
\hline Canadá & 528 & 11,1 & 12,4 \\
\hline Chile & 447 & 34,8 & 1,2 \\
\hline Corea & 516 & 14,4 & 10,6 \\
\hline Dinamarca & 502 & 15,9 & 7,0 \\
\hline Eslovaquia & 461 & 30,7 & 3,6 \\
\hline Eslovenia & 513 & 15,0 & 10,6 \\
\hline España & 493 & 18,3 & 5,0 \\
\hline Estados Unidos & 496 & 20,3 & 8,5 \\
\hline Estonia & 534 & 8,8 & 13,5 \\
\hline Finlandia & 531 & 11,5 & 14,3 \\
\hline Francia & 495 & 22,1 & 8,0 \\
\hline Grecia & 455 & 32,7 & 2,1 \\
\hline Holanda & 509 & 18,5 & 11,1 \\
\hline Hungría & 477 & 26,0 & 4,6 \\
\hline Irlanda & 503 & 15,3 & 7,1 \\
\hline Islandia & 473 & 25,3 & 3,8 \\
\hline Israel & 467 & 31,4 & 5,8 \\
\hline Italia & 481 & 23,2 & 4,1 \\
\hline Japón & 538 & 9,6 & 15,3 \\
\hline Letonia & 490 & 17,2 & 3,8 \\
\hline Luxemburgo & 483 & 25,9 & 6,9 \\
\hline México & 416 & 47,8 & 0,1 \\
\hline Nieva Zelanda & 513 & 17,4 & 12,8 \\
\hline Noruega & 498 & 18,7 & 8,0 \\
\hline Polonia & 501 & 16,3 & 7,3 \\
\hline Portugal & 501 & 17,4 & 7,4 \\
\hline Reino Unido & 509 & 17,4 & 10,9 \\
\hline República Checa & 493 & 20,7 & 7,3 \\
\hline Suecia & 493 & 21,6 & 8,5 \\
\hline Suiza & 506 & 18,5 & 9,8 \\
\hline Turquía & 425 & 44,5 & 0,3 \\
\hline OECD (media-34) & 493 & 21,3 & 7,7 \\
\hline $\mathrm{CV}$ & 0,055 & 0,401 & 0,492 \\
\hline
\end{tabular}

FUENTE: Informe PISA (2015). 


\section{GRÁFICO 2}

ALTO Y BAJO RENDIMIENTO EN ESPAÑA. DISTRIBUCIÓN PORCENTUAL (PISA 2015, CIENCIAS)

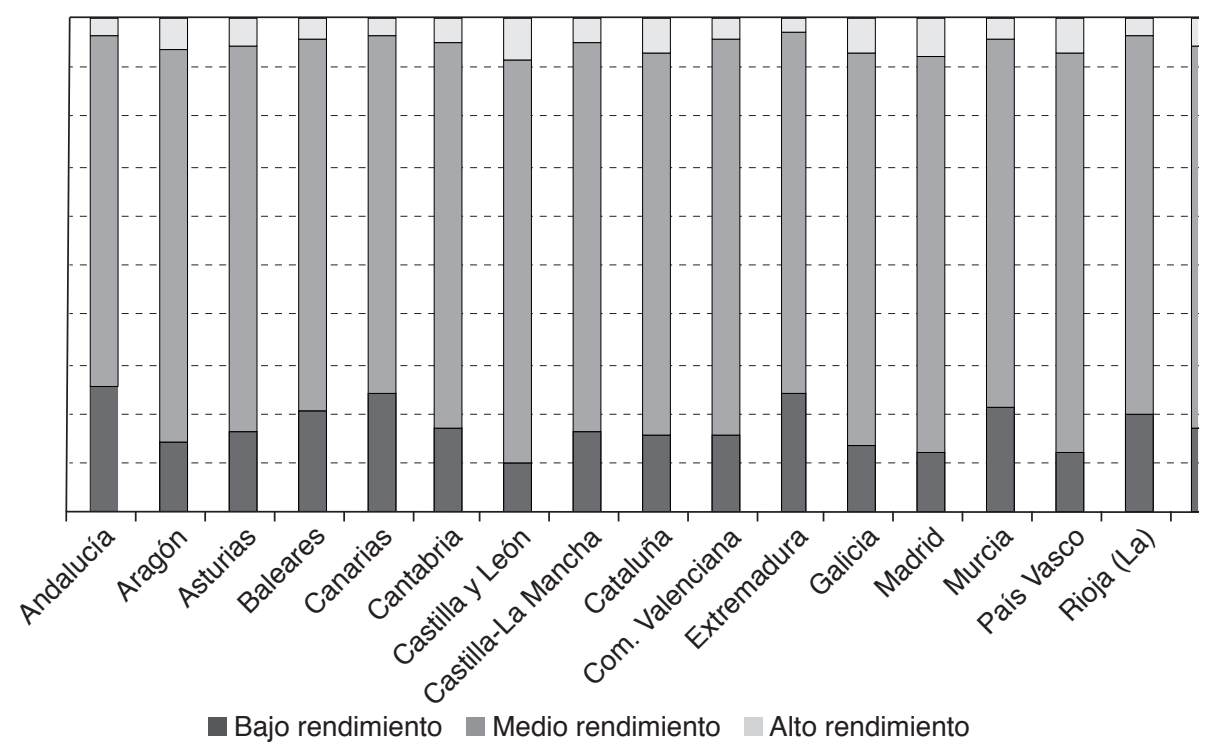

FUENTE: Ministerio de Educación a partir de Informe PISA (OCDE).

inspección ocular muestra algunos rasgos destacables, que luego veremos con mayor detalle. El primero, que el porcentaje de estudiantes con bajo rendimiento es mayor que el de estudiantes con alto rendimiento en todas las regiones. El segundo, que existe una gran diversidad de situaciones en las distintas comunidades. Y también que no hay una correlación clara entre nivel socioeconómico de la región y rendimiento educativo.

\section{Rendimiento}

La forma más elemental de medir el rendimiento es tomar los valores medios de los test de cada sociedad que ofrece el propio Informe PISA. Esta sencillez no es gratuita dado que al centrarnos en los valores medios ignoramos todos los otros aspectos relacionados con las diferencias existentes en las distribuciones. El Cuadro 3 proporciona los resultados relativos a las comunidades autónomas, donde se aprecia una clara división Norte-Sur, que presenta una baja correlación con los valores de renta per cápita o incluso de los niveles educativos de la población en edad de trabajar. Lo que indica que detrás de los resultados observados hay variables específicas de tipo idiosincrático que no se resumen en la riqueza de los hogares o la formación media de los adultos. Aspectos como la valoración social del conocimiento, la implicación 
CUADRO 3

VALORES MEDIOS DE LOS TEST PISA

2015 (CIENCIAS)

EN LAS COMUNIDADES

AUTÓNOMAS ESPAÑOLAS

\begin{tabular}{|l|c|}
\hline \multicolumn{1}{|c|}{ CCAA } & Puntuación \\
\hline Andalucía & 473 \\
\hline Aragón & 508 \\
\hline Asturias & 501 \\
\hline Baleares & 485 \\
\hline Canarias & 475 \\
\hline Cantabria & 496 \\
\hline Castilla León & 519 \\
\hline Castilla Mancha & 497 \\
\hline Cataluña & 504 \\
\hline Comunidad Valenciana & 494 \\
\hline Extremadura & 474 \\
\hline Galicia & 512 \\
\hline Madrid & 516 \\
\hline Murcia & 484 \\
\hline Navarra & 512 \\
\hline País Vasco & 483 \\
\hline Rioja & 498 \\
\hline Media & 496 \\
\hline CV & 0,0291 \\
\hline
\end{tabular}

FUENTE: Ministerio de Educación a partir de Informe PISA (OCDE).

de las familias en la educación, la calidad del profesorado y el reconocimiento de su labor, o la formación de expectativas juegan también un papel en la explicación de la diferencia de resultados.

La variabilidad de resultados en los valores medios del test es muy reducida, como acredita un coeficiente de variación inferior a 0,03. Sin embargo conviene advertir que la diferencia entre Castilla y León y Andalucía es de 46 puntos, lo que equivale a más de un año de escolarización. Hay que subrayar, no obstante, que las diferencias sustanciales entre comunidades no se refieren al rendimiento medio sino a la distribución de resultados entre los distintos niveles de competencia.

El Gráfico 3 describe las puntuaciones relativas de las comunidades, tomando como 100 la media de todas ellas, y ordenando estas de mayor a menor. 


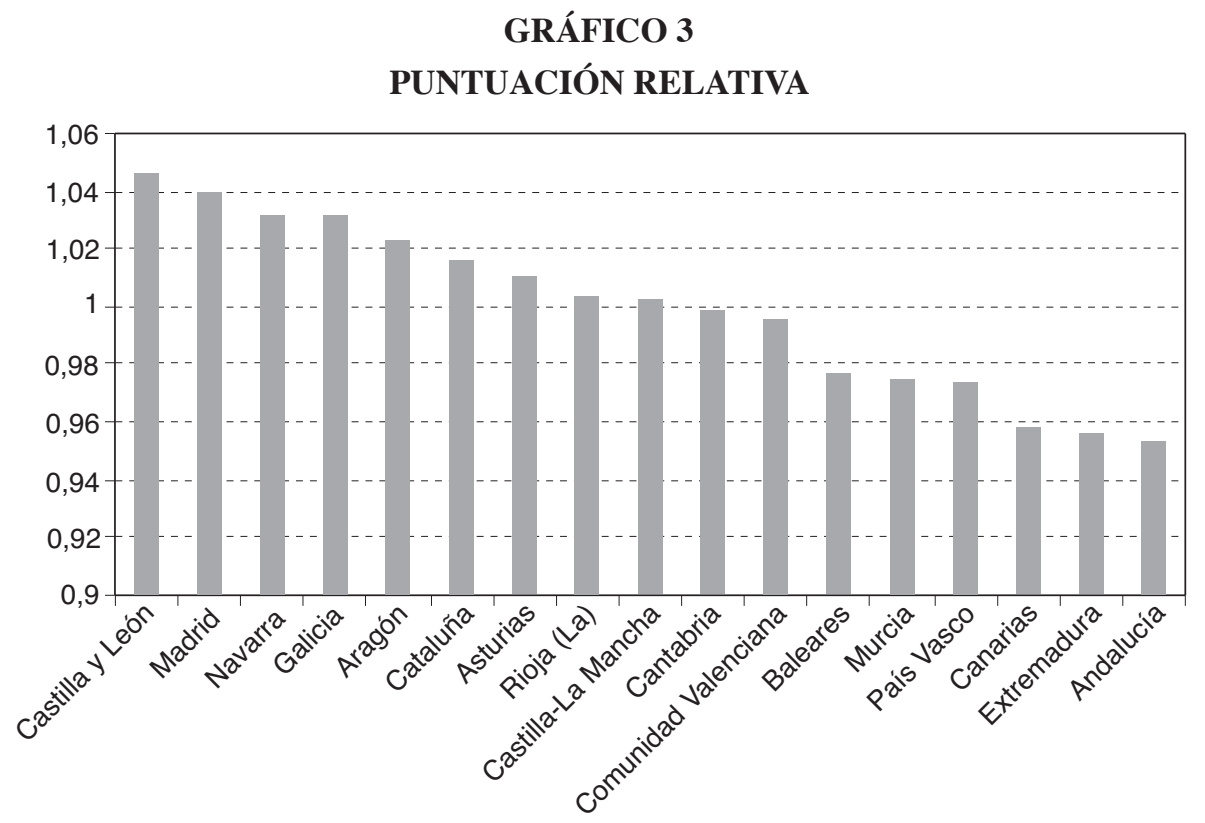

FUENTE: Ministerio de Educación a partir de Informe PISA (OCDE).

Observación: Martínez y Villar (2018) proponen una manera más rica de medir el rendimiento. Se trata de evaluarlo como una suma ponderada, con ponderadores dados por las correspondientes fracciones de población, de una función de los valores medios de los test en los siete diferentes niveles de rendimiento considerados. Esta función no es más que la ratio entre dicho valor medio para cada nivel de rendimiento y la media nacional, elevada al cuadrado. Es decir, para la región i y los niveles de rendimiento $j$,

$$
R_{i}=\sum_{j=1}^{7} \frac{n_{i j}}{n_{i}} \times\left(\frac{P_{i j}}{\mu}\right)^{2}
$$

donde $n$ hace referencia a la población, $P$ al valor medio del test del grupo correspondiente, y $\mu$ a la media del test en España. Con esta fórmula se da una importancia creciente a los valores por encima de la media nacional, y viceversa.

\section{Equidad}

La idea de «equidad» de los sistemas educativos está asociada al grado de dependencia de los resultados observados con respecto a las circunstancias de los 
individuos. Cuanto más independientes son los resultados de los estudiantes de su entorno familiar y social más equitativo es el sistema. Y viceversa. Esta idea, vinculada a la noción de igualdad de oportunidades (Roemer, 1998), es especialmente importante en este contexto en el que se miden los resultados de la educación obligatoria, que trata de garantizar un conjunto mínimo de conocimientos y habilidades para toda la población.

Hay diversas formas de aproximar la medición de la equidad de los sistemas educativos, pero prácticamente todas toman como referencia la relación que existe entre los resultados de los test y el Índice de Estatus Socioeconómico y Cultural (ISEC). Este indicador se usa para estimar el nivel cultural y socioeconómico de sus familias, que es la variable de entorno más importante, y combina la información sobre la educación de los padres y sus ocupaciones así como las posesiones en el hogar ${ }^{2}$. Existe una correlación positiva en todos los países entre este indicador y los resultados de los test, si bien la intensidad de la correlación es muy variada (lo que significa que hay otros factores importantes en la explicación de los resultados).

Una idea inicial del grado de equidad de los sistemas educativos puede obtenerse analizando la distribución de resultados en función del grupo social al que pertenecen los estudiantes. Para fijar ideas consideraremos aquí cuatro grupos sociales, los determinados por los cuartiles de la distribución del ISEC, y nos fijaremos en las proporciones de estudiantes de alto y bajo rendimiento en los extremos, cuartiles primero (familias menos favorecidas) y cuarto (familias con mayores recursos). El Cuadro 4 proporciona información sobre cómo varían estos porcentajes de estudiantes de alto y bajo rendimiento según su origen social, en los cuartiles primero y cuarto de la distribución del índice socioeconómico y cultural. Adviértase que la diversidad entre comunidades es del orden de 10 veces la diversidad de los resultados de los test, según el coeficiente de variación.

A partir de estos datos podemos visualizar el grado de dependencia de los resultados con respecto al origen social simplemente observando qué porcentaje del total de estudiantes de alto y bajo rendimiento provienen de cada uno de estos grupos sociales. El Cuadro 5 proporciona esta información. Tomemos el caso de Andalucía como ejemplo. Más del 37 por 100 de los estudiantes de bajo rendimiento provienen de familias en el primer cuartil del ISEC, mientras que poco más del 6 por 100 de los estudiantes de alto rendimiento provienen de este colectivo. La ratio 1/4 es una medida de discriminación que nos dice que hay 3,6 veces más estudiantes de bajo rendimiento que provienen del primer cuartil de los que provienen del cuarto cuartil.

${ }^{2}$ Este índice «combina la información sobre la educación de los padres y sus ocupaciones, así como las posesiones en el hogar. Se obtiene a partir de las siguientes variables: el índice internacional de estatus ocupacional del padre o la madre, el que sea más alto, convertido en años de escolarización; y el índice de posesiones en el hogar, obtenido a partir de preguntas a los estudiantes sobre si disponen de una mesa para estudiar en casa, una habitación independiente, un lugar tranquilo para estudiar, software educativo, conexión a internet, su propia calculadora, libros de literatura clásica y poesía, libros de arte (por ejemplo, pintura), libros que les ayuden en sus tareas escolares, un diccionario, lavaplatos, reproductor de DVD o video, tres cuestiones adicionales específicas de cada país, y el número de teléfonos móviles, televisores, ordenadores, coches y libros que hay en casa» (Ministerio de Educación Cultura y Deporte, 2016). 


\section{CUADRO 4}

ESTUDIANTES DE ALTO Y BAJO RENDIMIENTO EN CIENCIAS, PISA 2015 (TOTAL, PRIMER Y CUARTO CUARTIL DEL ISEC)

(En \%)

\begin{tabular}{|l|c|c|c|c|c|c|}
\hline \multirow{2}{*}{} & \multicolumn{2}{|c|}{ Total } & \multicolumn{2}{c|}{ Primer cuartil } & \multicolumn{2}{c|}{ Cuarto cuartil } \\
\cline { 2 - 7 } & $\begin{array}{c}\text { Bajo ren- } \\
\text { dimiento }\end{array}$ & $\begin{array}{c}\text { Alto ren- } \\
\text { dimiento }\end{array}$ & $\begin{array}{l}\text { Bajo ren- } \\
\text { dimiento }\end{array}$ & $\begin{array}{c}\text { Alto ren- } \\
\text { dimiento }\end{array}$ & $\begin{array}{c}\text { Bajo ren- } \\
\text { dimiento }\end{array}$ & $\begin{array}{c}\text { Alto ren- } \\
\text { dimiento }\end{array}$ \\
\hline Andalucía & 25,4 & 3,2 & 38,0 & 0,8 & 10,7 & 7,9 \\
\hline Aragón & 14,2 & 6,6 & 24,3 & 3,0 & 5,1 & 12,4 \\
\hline Asturias & 16,3 & 5,9 & 29,3 & 2,1 & 4,1 & 11,2 \\
\hline Baleares & 20,4 & 4,0 & 31,3 & 1,3 & 9,4 & 8,4 \\
\hline Canarias & 23,8 & 3,2 & 37,2 & 1,4 & 9,6 & 8,2 \\
\hline Cantabria & 17,3 & 4,8 & 27,8 & 2,4 & 6,3 & 10,2 \\
\hline Castilla y León & 10,2 & 8,5 & 16,5 & 3,9 & 5,1 & 15,0 \\
\hline Castilla-La Mancha & 16,2 & 5,0 & 25,0 & 1,9 & 6,1 & 10,1 \\
\hline Cataluña & 15,7 & 6,7 & 26,8 & 2,0 & 4,4 & 14,2 \\
\hline Com. Valenciana & 15,8 & 4,1 & 26,6 & 1,3 & 5,9 & 9,8 \\
\hline Extremadura & 24,2 & 3,1 & 38,2 & 1,0 & 11,5 & 6,3 \\
\hline Galicia & 13,4 & 7,4 & 20,1 & 3,3 & 5,7 & 12,9 \\
\hline Madrid & 12,2 & 7,6 & 23,9 & 3,1 & 3,3 & 13,6 \\
\hline Murcia & 21,1 & 3,9 & 38,1 & 1,4 & 7,2 & 8,6 \\
\hline Navarra & 11,9 & 6,8 & 21,6 & 2,7 & 4,0 & 13,9 \\
\hline País Vasco & 20,2 & 3,3 & 30,2 & 0,8 & 10,9 & 5,5 \\
\hline Rioja (La) & 17,0 & 5,9 & 31,5 & 1,9 & 7,6 & 11,5 \\
\hline Media & 17,4 & 5,3 & 28,6 & 2,0 & 6,9 & 10,6 \\
\hline Coef. de variación & 0,252 & 0,324 & 0,223 & 0,448 & 0,371 & 0,261 \\
\hline
\end{tabular}

FUENTE: Ministerio de Educación a partir de Informe PISA (OCDE).

En el otro extremo observamos que casi el 62 por 100 de los estudiantes con alto rendimiento provienen de las familias más favorecidas mientras que algo más del 10 por 100 de los estudiantes con bajo rendimiento provienen del cuarto cuartil del ISEC. En este caso la ratio 4/1 nos dice que hay casi 10 veces más estudiantes de alto rendimiento que provienen del cuarto cuartil de los que provienen del primer cuartil.

Tengamos en cuenta que en un sistema perfectamente equitativo, en el que no hubiera dependencia de los resultados del origen social, la distribución por niveles de rendimiento debería ser aproximadamente uniforme por cuartiles. Es decir, las ratios $1 / 4$ y $4 / 1$ debieran estar siempre en torno a 1 . En consecuencia, los valores de estas ratios nos permiten considerar dos tipos de discriminación en los sistemas 


\section{CUADRO 5}

PORCENTAJE DE ESTUDIANTES DEL PRIMER Y CUARTO CUARTIL ENTRE LOS DE BAJO Y ALTO RENDIMIENTO (CIENCIAS, PISA 2015) (En \%)

\begin{tabular}{|l|c|c|c|c|c|c|}
\hline & \multicolumn{3}{|c|}{ Estudiantes bajo rendimiento } & \multicolumn{2}{c|}{ Estudiantes alto rendimiento } \\
\cline { 2 - 7 } & $\begin{array}{c}\text { Primer } \\
\text { cuartil }\end{array}$ & $\begin{array}{c}\text { Cuarto } \\
\text { cuartil }\end{array}$ & Ratio 1/4 & $\begin{array}{c}\text { Primer } \\
\text { cuartil }\end{array}$ & $\begin{array}{c}\text { Cuarto } \\
\text { cuartil }\end{array}$ & Ratio 4/1 \\
\hline Andalucía & 37,40 & 10,50 & 3,6 & 6,30 & 61,70 & 9,8 \\
\hline Aragón & 42,80 & 9,00 & 4,8 & 11,40 & 47,00 & 4,1 \\
\hline Asturias & 44,90 & 6,30 & 7,1 & 8,90 & 47,50 & 5,3 \\
\hline Baleares & 38,40 & 11,50 & 3,3 & 8,10 & 52,50 & 6,5 \\
\hline Canarias & 39,10 & 10,10 & 3,9 & 10,90 & 64,10 & 5,9 \\
\hline Cantabria & 40,20 & 9,10 & 4,4 & 12,50 & 53,10 & 4,2 \\
\hline Castilla y León & 40,40 & 12,50 & 3,2 & 11,50 & 44,10 & 3,8 \\
\hline Castilla-La Mancha & 38,60 & 9,40 & 4,1 & 9,50 & 50,50 & 5,3 \\
\hline Cataluña & 42,70 & 7,00 & 6,1 & 7,50 & 53,00 & 7,1 \\
\hline Comunidad Valenciana & 42,10 & 9,30 & 4,5 & 7,90 & 59,80 & 7,6 \\
\hline Extremadura & 39,50 & 11,90 & 3,3 & 8,10 & 50,80 & 6,3 \\
\hline Galicia & 37,50 & 10,60 & 3,5 & 11,10 & 43,60 & 3,9 \\
\hline Madrid & 49,00 & 6,80 & 7,2 & 10,20 & 44,70 & 4,4 \\
\hline Murcia & 45,10 & 8,50 & 5,3 & 9,00 & 55,10 & 6,1 \\
\hline Navarra & 45,40 & 8,40 & 5,4 & 9,90 & 51,10 & 5,2 \\
\hline País Vasco & 37,40 & 13,50 & 2,8 & 6,10 & 41,70 & 6,8 \\
\hline Rioja (La) & 46,30 & 11,20 & 4,1 & 8,10 & 48,70 & 6,0 \\
\hline
\end{tabular}

FUENTE: Ministerio de Educación a partir de Informe PISA (OCDE).

educativos: Discriminación «por arriba», medida como el valor de la ratio 4/1 (porcentaje de estudiantes con alto rendimiento con origen familiar en el cuarto cuartil con relación a los provenientes del primer cuartil), y discriminación «por abajo» (porcentaje de estudiantes con bajo rendimiento procedentes del primer cuartil en relación a los del cuarto). Si comparamos Andalucía con Madrid, por ejemplo, observamos que la discriminación «por abajo» es más intensa en Madrid, mientras que la discriminación «por arriba» es mayor en Andalucía. La variabilidad de estos tipos de discriminación entre las regiones es muy alta, con un coeficiente de variación de 0,285 para el caso de la discriminación por abajo y un coeficiente de 0,259 para el caso de la discriminación por arriba. Es también interesante observar que existe una correlación muy baja y negativa entre ambos tipos de discriminación (un coeficiente de correlación de $-0,19)$. 
Los datos muestran una gran variedad de comportamientos entre las regiones, con importantes diferencias sobre el tipo de discriminación que existe. Asturias, Cataluña y Madrid son las regiones con mayores niveles de discriminación por abajo, mientras que Andalucía, Cataluña y Comunidad Valenciana son las regiones con mayores niveles de discriminación por arriba. Estos elementales indicadores nos proporcionan, pues, información sobre el tipo de desigualdad de oportunidades de mayor entidad en las distintas comunidades autónomas y, por tanto, el tipo de objetivos que deben centrar la acción de cara a mejorar la equidad (para un estudio desde esta perspectiva en el caso de América Latina, véase Villar y Zoido, 2016).

Una forma alternativa y más compacta de medir el grado de equidad de un sistema educativo, que resulta habitual en la literatura, es mediante el coeficiente de determinación, $R^{2}$ (el cuadrado del coeficiente de correlación entre los valores del test y los del ISEC). Se trata de una medida comprendida entre 0 y 1 que nos dice cuál es el porcentaje de la varianza compartida entre las dos series de datos. Esta variable proporciona una medida adecuada de la dependencia de los resultados de los individuos de sus condiciones socioeconómicas y culturales y puede, por tanto, tomarse como un indicador de «desigualdad de oportunidades» (para una discusión en profundidad sobre la idoneidad de esta medida, véase Ferreira y Ginoux, 2011).

El Cuadro 6 proporciona los datos de la correlación entre resultados y origen familiar para las distintas comunidades. El coeficiente de variación (CV) indica una notable diversidad en el grado de inequidad de los sistemas educativos.

El Gráfico 4 proporciona esta misma información en términos relativos, tomando la media de inequidad igual a 100 , y ordenando las comunidades de menor a mayor desigualdad de oportunidades. Andalucía, Asturias, Canarias, Cataluña, Madrid, Murcia y La Rioja muestran valores de desigualdad de oportunidades que superan en más de 10 puntos porcentuales a la media. Por el contrario, Baleares, Cantabria, Castilla y León, Galicia y País Vasco son las comunidades con mayor igualdad de oportunidades.

Es interesante observar que hay una correlación baja pero negativa $(R=-0,14)$ entre rendimiento e inequidad. Esto significa que conseguir mayores niveles de igualdad de oportunidades no significa renunciar a rendimientos más altos. 


\section{CUADRO 6 \\ DEPENDENCIA DE LOS \\ RESULTADOS EDUCATIVOS DE LAS \\ CONDICIONES SOCIOECONÓMICAS \\ Y CULTURALES (ISEC) (PISA 2015 \\ CIENCIAS)}

\begin{tabular}{|l|l|}
\hline \multicolumn{1}{|c|}{$\begin{array}{c}\text { Comunidades } \\
\text { Autónomas }\end{array}$} & $\boldsymbol{R}^{\mathbf{2}}$ \\
\hline Andalucía & 0,131 \\
\hline Aragón & 0,124 \\
\hline Asturias & 0,156 \\
\hline Baleares & 0,090 \\
\hline Canarias & 0,127 \\
\hline Cantabria & 0,089 \\
\hline Castilla y León & 0,070 \\
\hline Castilla-La Mancha & 0,112 \\
\hline Cataluña & 0,136 \\
\hline Comunidad Valenciana & 0,108 \\
\hline Extremadura & 0,108 \\
\hline Galicia & 0,059 \\
\hline Madrid & 0,144 \\
\hline Murcia & 0,170 \\
\hline Navarra & 0,123 \\
\hline País Vasco & 0,076 \\
\hline Rioja (La) & 0,128 \\
\hline CV & 0,257 \\
\hline
\end{tabular}

FUENTE: Ministerio de Educación a partir de Informe PISA (OCDE). 


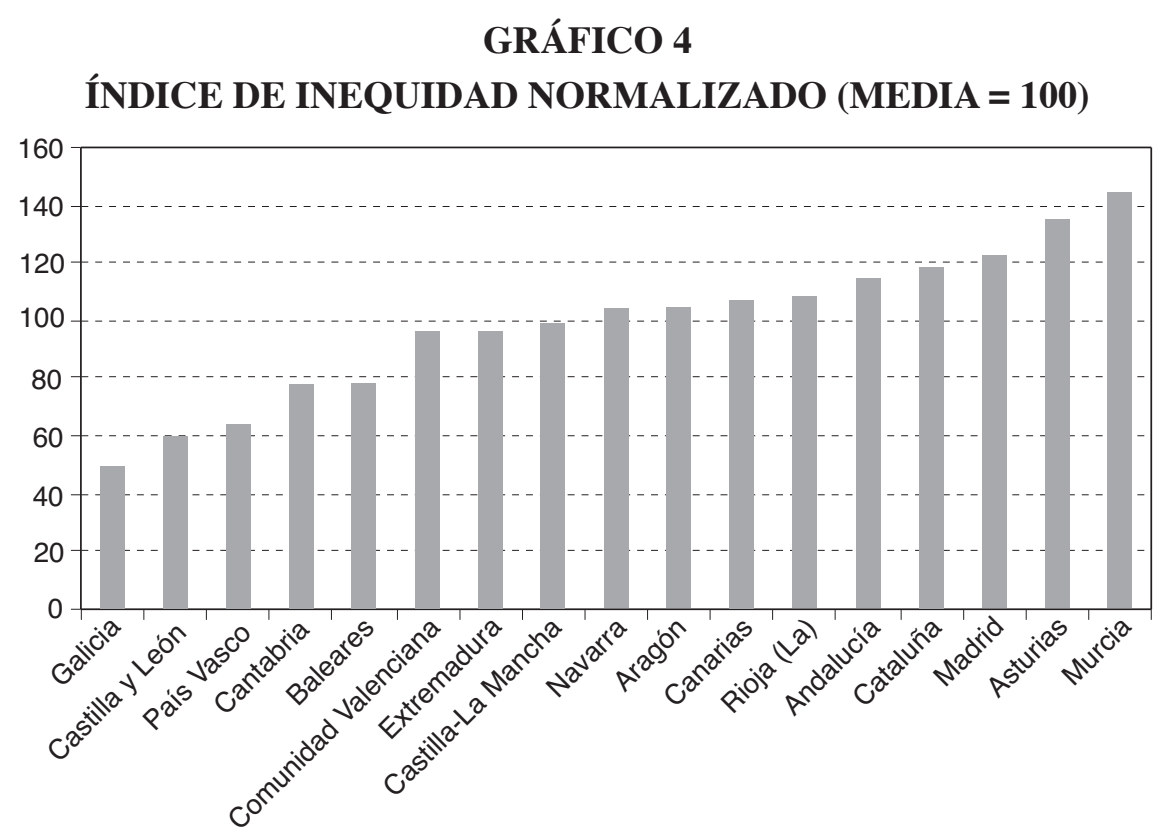

FUENTE: Ministerio de Educación a partir de Informe PISA (OCDE).

\section{Calidad: excelencia e inclusión}

La idea de calidad admite toda una variedad de interpretaciones dado el carácter multifacético de este concepto. Aquí vincularemos la idea de calidad a dos únicos elementos medibles mediante variables contenidas en el Informe PISA: la «excelencia» (entendida como la proporción de estudiantes con alto rendimiento) y la «inclusión» (entendida como la proporción de estudiantes que superan el mínimo imprescindible para poder desenvolverse razonablemente en la vida). Ciertamente otras interpretaciones son posibles pero esta será la que adoptaremos aquí.

Para medir la excelencia, que denotamos por $A$, tomamos como variable el porcentaje de estudiantes de alto rendimiento (niveles 5 y 6). Para medir la inclusión, que denotamos por $N$, tomamos la proporción de estudiantes que no son de bajo rendimiento (aquellos que alcanzan los niveles 2 o superior). Adviértase que $N=1-B$, donde $B$ es la fracción de estudiantes de bajo rendimiento.

Nuestro índice de calidad no es más que el producto de estas dos variables:

$$
C=A(1-B)
$$

Este índice $C$ puede entenderse como una medida de excelencia, $A$, deflactada por una medida de «fracaso», $B$, o bien como una medida de inclusión, $N$, potenciada por una medida de excelencia. 
El Cuadro 7 ofrece los datos relativos a estas variables. El Índice de Calidad viene expresado como porcentaje de la media de las comunidades, para dar una idea relativa de la situación de las mismas. Adviértase que las comunidades autónomas españoles difieren notablemente en este aspecto, con un coeficiente de variación 10 veces el correspondiente al rendimiento. El rango de variación del índice de calidad es amplísimo, del 172 de Castilla y León al 53 de Extremadura.

El Gráfico 5 ilustra la distribución de este indicador de calidad ordenando las comunidades de mayor a menor valor.

\section{CUADRO 7}

EXCELENCIA, INCLUSIÓN Y CALIDAD (PISA 2015, CIENCIAS)

\begin{tabular}{|l|c|c|c|}
\hline & Excelencia & Inclusión & $\begin{array}{c}\text { Índice de calidad } \\
\text { (relativo) }(\%)\end{array}$ \\
\hline Andalucía & 0,032 & 0,746 & 53,3 \\
\hline Aragón & 0,066 & 0,858 & 128,2 \\
\hline Asturias & 0,059 & 0,836 & 110,9 \\
\hline Baleares & 0,040 & 0,796 & 71,0 \\
\hline Canarias & 0,032 & 0,762 & 55,3 \\
\hline Cantabria & 0,048 & 0,827 & 88,6 \\
\hline Castilla y León & 0,085 & 0,898 & 172,1 \\
\hline Castilla-La Mancha & 0,050 & 0,838 & 94,4 \\
\hline Cataluña & 0,067 & 0,843 & 126,8 \\
\hline Comunidad Valenciana & 0,041 & 0,842 & 78,3 \\
\hline Extremadura & 0,031 & 0,757 & 52,8 \\
\hline Galicia & 0,074 & 0,866 & 143,7 \\
\hline Madrid & 0,076 & 0,878 & 150,8 \\
\hline Murcia & 0,039 & 0,789 & 69,7 \\
\hline Navarra & 0,068 & 0,881 & 135,5 \\
\hline País Vasco & 0,032 & 0,798 & 58,4 \\
\hline Rioja (La) & 0,059 & 0,830 & 109,9 \\
\hline Media & 0,053 & 0,8 & 100 \\
\hline Coeficiente de Variación & 0,324 & 0,053 & 0,369 \\
\hline
\end{tabular}

FUENTE: Ministerio de Educación a partir de Informe PISA (OCDE). 


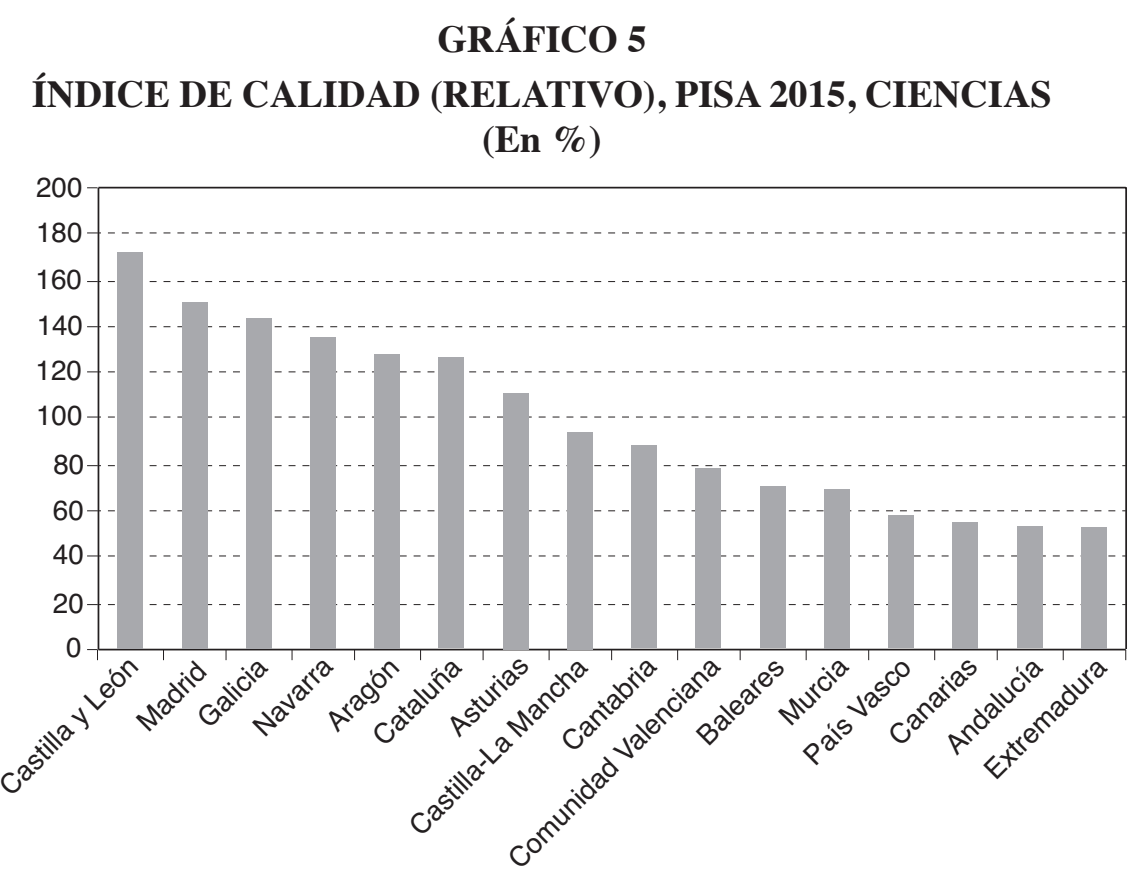

FUENTE: Ministerio de Educación a partir de Informe PISA (OCDE).

Claramente las diferencias en la calidad de los sistemas educativos son las más importantes de las que se observan en las comunidades autónomas españolas.

\section{Comentarios finales}

En este trabajo hemos presentado una análisis de los resultados de PISA en España y sus comunidades autónomas, teniendo en cuenta tres diferentes aspectos, el rendimiento, la equidad y la calidad. Esta aproximación trata de evitar el reduccionismo de utilizar los valores medios de los test, dadas las grandes diferencias en las distribuciones de resultados por niveles de rendimiento.

Una de las primeras conclusiones del estudio es que la escasa variabilidad de los valores medios entre las regiones españolas esconde una notable diferencia de situaciones en cuanto a la distribución de estudiantes por niveles de rendimiento. Y también que estos niveles de rendimiento están fuertemente ligadas al perfil socioeconómico y cultural de las familias de origen de los estudiantes. Dicho en otros términos, los problemas esenciales que apuntan los resultados de PISA con respecto al sistema educativo español se refieren a: $a$ ) las enormes diferencias que encontramos entre las regiones, en particular en lo relativo a calidad de los sistemas educativos, que se refleja en las proporciones de estudiantes de alto y bajo rendimiento; b) la reducida proporción de estudiantes de alto rendimiento en nuestro país en 
comparación con la OCDE; y c) la falta de equidad: La excesiva dependencia del alto y bajo rendimiento del entorno familiar, lo que indica un insuficiente logro de la igualdad de oportunidades.

Quiénes son sus padres y en qué región se educa afecta mucho al valor esperado de la educación que un joven alcanzará. Y con ello al tipo de empleo que obtenga y al nivel salarial. En el informe de la OCDE que acaba de aparecer, Panorama de la Educación 2018 (OCDE, 2018), se hace hincapié en la existencia de una «trampa intergeneracional» que se refleja en altas tasas de abandono escolar temprano que se concentran en jóvenes cuyos padres tienen bajos niveles educativos. Más de la mitad de los jóvenes que no terminan la educación secundaria provienen de familias con un bajo nivel educativo. La escuela parece estar dejando de proporcionar ese «ascensor social» que antaño permitía el progreso de los menos favorecidos merced a la formación y el esfuerzo.

Conviene recordar, a riesgo de caer en una obviedad, que la educación no es solo producto de la escuela sino del entorno familiar y social, de los patrones culturales, de la implicación social en los procesos de aprendizaje, la valoración de la formación, de las aspiraciones de los jóvenes y su entorno, etc. Se trata de elementos difíciles de cuantificar y aún más difíciles de modificar mediante políticas públicas, pero que están detrás de las diferencias de resultados que persisten entre las distintas comunidades autónomas, una vez descontados los efectos de los diferentes grados de desarrollo (renta per cápita, años medios de escolarización, gasto educativo, etc.).

El capital cultural de las familias es un elemento clave en el rendimiento de los estudiantes, más que la riqueza o el tipo de actividad profesional. Un joven que ha adquirido el hábito de lectura desde la niñez, que está inmerso en un entorno en el que se habla de lo que ocurre en el mundo, donde se fomenta la curiosidad, el interés por el arte, donde se discuten y razonan las decisiones, se generan expectativas ambiciosas, etc., tiene un punto de partida que le facilitará alcanzar altos niveles formativos. Y lo contrario ocurre para quien no se encuentra en un entorno rico culturalmente. Es quizá en estos aspectos donde la escuela debe tratar de compensar las diferencias sociales lo antes posible. El alto porcentaje de cobertura de educación pre-escolar puede ser un elemento importante de suavización de las diferencias en el capital cultural de las familias.

Es significativo que el número de libros que hay en el hogar es una variable explicativa más importante que la renta o la educación de los padres.

\section{Referencias bibliográficas}

[1] ACEMOGLU, D. y ROBINSON, J. A. (2013). Why Nations Fail: The Origins of Power, Prosperity, and Poverty, Broadway Business.

[2] FERREIRA, F. H. G. y GINOUX, J. (2011). «The Measurement of Educational Inequality: Achievement and Opportunity». IZA Working Paper núm. 6161. 
[3] MARTÍNEZ, R. y VILLAR, A. (2018). «Multilevel Proficiency Comparisons with an Application to Educational Outcomes in PISA». Working Paper núm. 1804, Universidad Pablo de Olavide.

[4] MINISTERIO DE EDUCACIÓN, CULTURA Y DEPORTE (2016). PISA 2015. Programa para la Evaluación Internacional de los Alumnos. Informe español.

[5] OCDE (2018). Panorama de la Educación. Informe español. Ministerio de Educación y Formación Profesional.

[6] ROEMER, JOHN E. (1998). Equality of opportunity. Cambridge, Mass., Harvard University Press.

[7] VILLAR, A. (2013). «The Educational Development Index». Modern Economy, vol 4, 403-411.

[8] VILLAR, A. y ZOIDO, P. (2016). «Challenges to quality and equity in educational performance for Latin America, a PISA 2012 perspective». RELIEVE, 22 (1), M9. DOI: http://dx.doi.org/10.7203/relieve.22.1.8273. 